\title{
Air Bacteriology of Operation Theatres and Intensive Care Units from a Tertiary Care Hospital in New Delhi
}

\author{
Patel Pritikumari, Shalini Malhotra*, Saloni Garg, Renu Yadav, NK Bhatia, Nandini Duggal \\ Department of Microbiology, PGIMER, Dr. RML Hospital, New Delhi, India \\ Corresponding Author: Shalini Malhotra; drshalinimalhotra@yahoo.com
}

Received 07 November 2019;

Accepted 21 November 2019;

Published 25 November 2019

\begin{abstract}
Background: Hospital-associated infections are a major cause of patient morbidity and mortality. Microbiological contamination of air in the operation theaters (OTs) and Intensive care units (ICUs) is a major risk factor for surgical site and other hospital-associated infections. The aim of the study was to determine the microbial contamination of air in the OTs and ICUs of a tertiary care hospital in north India. Methods: The study was conducted in the department of microbiology, Dr. RML hospital \& PGIMER, New Delhi. Air samples were collected from 9 OTs and 4 ICUs by settle plate method between January 2017-June 2018. Bacterial species were isolated and identified by conventional methods. Results: A total of 630 samples were collected from environment of various OTs and ICUs. Out of these, 130 (20\%) plates showed bacterial growth and yielded 380 isolates. Emergency OT and ECS OT showed highest bacterial CFU per plate. The most common isolate was Bacillus species $472(75 \%)$ followed by micrococcus 352(56\%), Staphylococcus aureus $6(1 \%)$. Conclusion: Routine surveillance of OT and ICU air in conventional OTs where there is no control on air quality, meticulous cleaning and strict adherence to OT and ICU protocol are essential steps for the prevention of hospital acquired infections. Further, the hospitals need to develop and implement processes for good infection control practices
\end{abstract}

Keywords: Hospital-associated infections, Air bacteriology, Bacterial CFU per plate, OT and ICU air

\section{Introduction}

Operation theaters (OTs) and intensive care units (ICU) are the workplaces that need the highest hygiene standards, since these are the places where the most critically ill patients are treated ${ }^{[1]}$. Bacterial contamination of air in OTs and ICUs is a major risk factor for hospital-associated infections (HAI) like surgical site infections ${ }^{[2]}$. This is because bacteria remain suspended the air as aerosols. Enclosed environment as present in OTs and ICUs confine aerosols and allow them to build up to infectious levels ${ }^{[3]}$, thus putting patients at a greater risk of HAI. It is recommended that for conventional OTs, the biological load should not exceed 35 Bacteria Carrying Particles (bcp) per $\mathrm{m}^{3}$ in an empty OT and 180 $\mathrm{bcp} / \mathrm{m}^{3}$ during an operation ${ }^{[4]}$.

Microbiological testing of air is required to detect bacterial flora of hospital air ${ }^{[5]}$. The estimation of the bacterial load in air can be used as an index for the cleanliness of the environment as well as a source of HAI ${ }^{[6]}$.

Purpose of this study was to find out the prevalence of spectrum of bacterial flora in OTs to find out the frequency of contamination from various sites in OTs.

\section{Materials and Method}

www.ijirms.in
Air samples can be collected in two ways:

\section{1) Active air sampling}

In active air sampling, a sampling device draws a known volume of air through or over a particle collection device which can be a liquid or a solid culture media or a nitrocellulose membrane and the quantity of microorganism present is measured in bcp/ m32) Passive air sampling (the settle plates)

The present reterospective study was conducted in the Department of Microbiology, Dr. Ram Manohar Lohia Hospital and Post Graduate Institute of Medical Education and Research, New Delhi, India. Air samples were collected from all working conventional OTs and ICUs by settle plate method from January 2015 to December 2016[10 OTs and4 ICUs].

The settle plate method was a popular and valid method for assessing bacterial contamination of air. The "settle plates" are standard culture media plates, which are exposed to the air for a given time to collect aerosols which "sediment" out and are then incubated. The principle behind settle plates is that most microorganisms in air are associated with physical particles which are large enough to settle out of the air-stream due to gravity. Results are expressed in CFU/plate/time or in CFU/m2/hour ${ }^{[7]}$.

Blood agar plates were kept open in the OTs and ICUs at a height of $1 \mathrm{~m}$ and $1 \mathrm{~m}$ from the walls of the rooms. Sampling time 
was 1hour. All the blood agar plates were labeled properly and immediately transported to the Microbiology laboratory and incubated at $37^{\circ} \mathrm{C}$ under aerobic condition for 24 hours. The microbial air contamination was measured by counting the number of CFU/ settle plate of air.

Isolation and identification of isolates were done as per standard guidelines. All isolates were divided into two broad categories: 1) Environmental Contaminant e.g. Micrococcus, Coagulase Negative Staphylococcus (CONS), Bacillus sp. 2) Pathogens e. g. Staphylococcus aureus, Gram Negative Bacilli.

\section{Results}

The number of colonies per settle plate from all OTs ranged from 10 to more than 100. Maximum plates (493 blood agar plates) had colonies ranged between 10-50 colonies per plate, followed by 79 plates 51-100 colonies per plate, 34 settle plates showed more than 100 colonies and 19 plates showed less than 10 colonies per plate as shown in Figure 1.

\section{Colonies per Settle Plate}

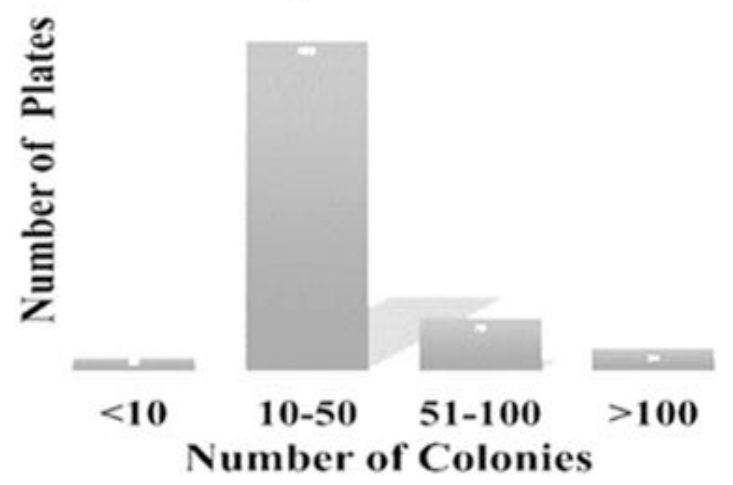

Figure 1: Colonies per settle plate

A total of 630 settle plates were collected. Out of these, 625 (99.2\%) plates showed growth of non-pathogenic bacteria. Emergency OT \& Emergency Care Services OT (ECS-OT) had the highest number of settle plates with growth followed by Minor OT, Maternity Nursing Home Labor Room (MNHLR), Cath lab, Trauma OT (TOT), Emergency services 4th floor. Renal Transplant ICU (RT-ICU) and Trauma ICU had minimum number of settle plates with bacterial growth. The most common isolates were ABS [aerobic spore bearers] 472 (75\%) followed by Micrococcus 352 (56\%), Coagulase Negative Staphylococcus Species $6(0.9 \%)$ as shown in Figure 2

\section{Number Of Settle Plates With Growth}

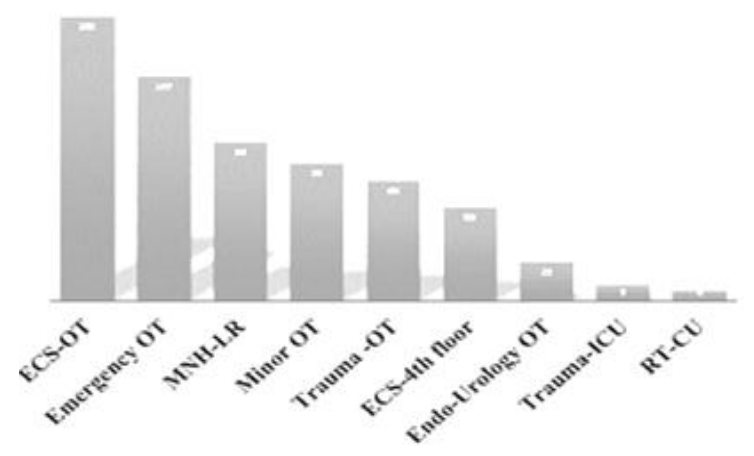

Figure 2: Number of settle plates with growth
ASBs in 472 plates (contaminants) and micrococci 352 plates (normal flora) were most common isolates followed by CONS 6 plates, (pathogens) as shown in Figure 3.

\section{Spectrum of flora from air ASB [472] \\ CONS \\ [06]}

Micrococcus [352]

Figure 3: Spectrum of flora from air

\section{Discussion}

The microbiological quality of air reflects the hygienic condition of operating room. Since air is an important vehicle for transmission of many pathogenic organisms, it is important to detect the number of bacteria carrying particles in operation theaters which is influenced by the factors besides with ventilation.

In our study, Emergency OT \& Emergency Care Services OT (ECS-OT) had highest number of settle plates with growth followed by Minor OT, Maternity Nursing Home Labor Room (MNHLR), Cath lab, Trauma OT (TOT), Emergency services 4th floor. Higher air contamination rates in Emergency OT \& Emergency Care Services OT (ECS-OT) observed could be due to many factors including unplanned emergency surgeries leading to decreased adherence to restricted entry in that area.

Renal Transplant ICU (RT-ICU) and Trauma ICU had minimum number of settle plates with bacterial growth because minimum occupancy at any time inside the OT is allowed. Anjali K et al, Mir RF et al, Kiranmai et al and Javed et al also reported in their study that ophthalmology OT and ENT OT was the one with least air contamination. ${ }^{[2],[8]-[10]}$

Corrective actions to take were advised in OT showing significant growth

1. Cleaning of OT with detergent and disinfectant after removal of dust on all surfaces by wet mopping.

2. Cleaning OT tables, lamps, trolleys with recommended disinfectant.

3. Fogging of OT using non-toxic disinfectant like Hydrogen peroxide (H2O2) / Super oxidized water etc. In conventional OT.

4. Restrict entry of personnel in OT and wear requisite PPE inside OT complex.

\section{Conclusion}

Settle plate method used to be a simple and effective way of detecting the microbiological contamination of OT and ICU air in conventional OTs and is not recommended any more. Meticulous cleaning and strict adherence to OT and ICU protocols are however essential steps for the prevention of HAI. The hospitals need to develop new age modular OTs with laminar airflow \& HEPA filters and implement safe infection control practices in all the areas of hospital including ICUs \& OTs. In such OTs, surveillance 
of air culture using air sampler/ Settle plate is not required and only controlled air changes, Positive pressure maintenance and proper maintenance of quality of HEPA filters is recommended.

The corrective actions can be advised in OT to improve quality of air like minimum 20 numbers of air changes per hour, 25-35 feet per minute air velocity, maintaining positive pressure, right temperature and humidity, dedicated air handling unit for each OT, with wall, floor and ceiling should be anti-static and made up of nonporous, smooth, seamless material, antibacterial antifungal painted. There should be minimum occupancy of persons at any time inside the OT.

Strengthening surveillance of HAI and laboratory capacity will surely enhance infection prevention and control. Routine sampling is strongly recommended for increasing awareness to identify and control all possible sources and types of infections. Settle plate's method for air is considered as crude method but in a limited resource setup these methods earlier proved to be more valuable in detecting the contamination level. Settle plates method had certain advantages like it was inexpensive, easy use, did not require any special equipment, gave information about qualitative analysis of airborne microorganisms and the data detected underlying trends in airborne contamination and provided early warning of problems. It helped in directly monitoring airborne contamination of specific surfaces. In future, more and more modular OTs with maintained air quality standards need to be adopted which will be very helpful as infection control measures.

\section{References}

[1] Ulger F, Esen S, Dilek A, Yanik K, Gunaydin M, Leblebicioglu H, "Are we aware how contaminated our mobile phones with nosocomial pathogens?," Annals of Clinical Microbiology and Antimicrobials, 8(7), 2009.

[2] Kiranmai S, Madhavi K, "Microbiological surveillance of operation theatres, intensive care units and labor room of a teaching hospital in Telangana, India," International
Journal of Research in Medical Sciences, 4, pp. 52565260, 2016.

[3] Landrin A, Bissery A, Kac G, "Monitoring air sampling in operating theatres: can particle counting replace microbiological sampling?' J Hosp Infect, 61, pp. 27-29, 2005.

[4] Arrowsmith LW, "Air sampling in operating theatres," Journal Hospital Infection, 6(3), pp. 352-353, 1985.

[5] ME Osman ME et al. "A study on microbiological contamination on air quality in hospitals in Egypt." Indoor and Built Environment, 27(7), pp. 953-968, 2017.

[6] Sehulster LM, Chinn RYW, Arduino MJ, Carpenter J, Donlan R, Ashford D, Besser R, Fields B, McNeil MM, Whitney C, Wong S, Juranek D, Cleveland J, "Guidelines for environmental infection control in health-care facilities. Recommendations from CDC and the Healthcare Infection Control Practices Advisory Committee (HICPAC). Chicago IL; American Society for Healthcare Engineering/American Hospital Association, 2004.

[7] Whyte W, "Sterility assurance and models for assessing airborne bacterial contamination," Journal of Parenteral Science and Technology, 40, pp. 188-197, 1986.

[8] Anjali K, Anamika V, Mrithunjay K, Dalal A S, Amritesh K, "Environmental microbiological surveillance of operation theatres in a tertiary care hospital," International Journal of Current Research, 7(03), pp. 13977-13980, 2015.

[9] Mir RF, Singh VA, Shinu P, "Pre-and post fumigation bacteriological profile of various operation theatres in MMIMSR-a three years retrospective study," Journal of pharmaceutical and biomedical sciences, 36(36), pp.1887-1891, 2013.

[10] Javed I, Hafeez R, Zubair M, Anwar M, Tayyib M, Husnain S, "Microbiological surveillance of operation theatres and ICUs of a teaching hospital, Lahore," Biomedica, 24, pp.99-102, 2008. 